\title{
Four-Wave Mixing Based 10-Gb/s Tunable Wavelength Conversion Using a Holey Fiber With a High SBS Threshold
}

\author{
Ju Han Lee, Walter Belardi, Kentaro Furusawa, Periklis Petropoulos, Zulfadzli Yusoff, Tanya M. Monro, and \\ David J. Richardson
}

\begin{abstract}
We demonstrate a four-wave-mixing based wavelength converter using a 15-m highly nonlinear holey fiber (HF) with a high stimulated Brillouin scattering (SBS) threshold. Error-free efficient wavelength conversion of $10-\mathrm{Gb} / \mathrm{s}$ nonreturn-to-zero signal over a $\sim 10$-nm bandwidth is reliably achieved. Our 15-m HF has a nonlinearity coefficient $\gamma \sim 70( \pm 10)$ $\mathrm{W}^{-1} \cdot \mathrm{km}^{-1}$ and an SBS threshold of more than $130 \mathrm{~mW}$. The high SBS threshold is achieved by applying structural variation to the HF along its length during the fabrication process. No modulational-instability-induced intensity noise on the converted signals is observed due to the normal dispersion of the HF.
\end{abstract}

Index Terms-Gratings, nonlinear optics, optical fiber communication, optical fiber devices, optical signal processing.

\section{INTRODUCTION}

$\mathbf{H}$ OLEY FIBER (HF) technology has advanced significantly in recent years and has resulted in the production of high quality of HFs with low loss [1] and ultrahigh nonlinearity [2]. HFs with an effective nonlinearity $10 \sim 100$ times higher than standard single-mode silica fibers can be achieved due to the large refractive index contrast between silica and air. Using such fibers, it thus becomes possible to implement nonlinear telecommunication devices with reduced length/power requirements relative to similar devices based on conventional optical fiber. A number of nonlinear HF-based devices have already been demonstrated including amongst others a Raman amplifier [3], a nonlinear thresholding device for optical code-division multiple-access systems [4], and a high-speed optical time-division multiplexing demultiplexer [5].

A high-speed wavelength converter is likely to be an essential nonlinear device within high capacity all-optical wavelength-division-multiplexing (WDM) networks, and there is, thus, considerable interest in the development of practical wavelength converters. We recently investigated the applicability of HF technology to this area and demonstrated a high-performance HF-based WDM wavelength converter using a cross-phase modulation (XPM) and filtering approach [6]. Error-free and almost penalty-free conversion operation was readily achieved in the experiments. An alternative, and more flexible approach, is, however, based on four-wave

Manuscript received September 27, 2002; revised December 2, 2002.

The authors are with the Optoelectronics Research Centre, University of Southampton, Highfield, Southampton, SO17 1BJ, U.K. (e-mail: jhl@orc.soton.ac.uk).

Digital Object Identifier 10.1109/LPT.2002.807928

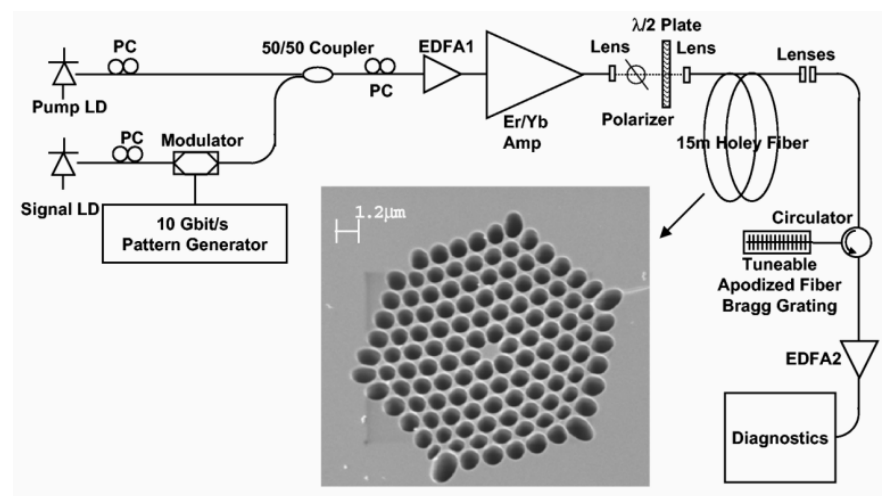

Fig. 1. Experimental setup. Inset: Cross-sectional SEM image of the high SBS threshold HF used within these experiments.

mixing (FWM). FWM is particularly attractive for wavelength conversion due to its relative transparency to both bit rate and modulation format. Highly efficient, broad-band wavelength conversion based on FWM in optical fiber requires high nonlinearity, low dispersion, a low dispersion slope, and a short fiber length so as to reduce the phase mismatch between the interacting waves [7], [8]. The reduction of stimulated Brillouin scattering (SBS) effects is a key issue in such devices and is required to suppress SBS-induced pump power loss [9].

In this letter, we demonstrate FWM-based tunable conversion using a highly nonlinear HF with a high SBS threshold. The high SBS threshold is obtained due to broadening of the Brillouin lineshape through structural variation along the fiber length [10]. Our 15-m HF has a nonlinearity coefficient $\gamma \sim 70( \pm 10) \mathrm{W}^{-1}$. $\mathrm{km}^{-1}$. The measured SBS threshold is more than $130 \mathrm{~mW}$ which is over three times higher than that of a uniform pure silica fiber. Error-free wavelength conversion over a $\sim 10$-nm bandwidth of a $10-\mathrm{Gb} / \mathrm{s}$ nonreturn-to-zero (NRZ) data rate is obtained with good efficiency. Modulation-instability-related amplitude noise associated with the wavelength conversion process is avoided by ensuring that the HF has normal dispersion [11].

\section{EXPERIMENTAL SETUP AND RESULTS}

The schematic of our experiment is shown in Fig. 1. Both the pump and the signal beams were derived from tunable continuous-wave (CW) external cavity lasers operating in the range $1530 \sim 1580 \mathrm{~nm}$. The signal beam at a fixed wavelength of $1550 \mathrm{~nm}$ was modulated with a $2^{15}-1$-pseudorandom data sequence at a data rate of $10 \mathrm{~Gb} / \mathrm{s}$ and then combined with the pump beam using a 50/50 coupler. The two beams were then 
amplified using an $\mathrm{Er}-\mathrm{Yb}$ amplifier with a maximum saturated power of $\sim 700 \mathrm{~mW}$ and then lens coupled into our 15-m-long HF with a coupling efficiency of $\sim 20 \%$. Polarization controllers (PCs), a polarizer, and a $\lambda / 2$ plate were used to ensure that both beams were launched onto a single polarization axis of our polarization maintaining HF, which had a beat length of $\sim 0.5 \mathrm{~mm}$ and a polarization extinction ratio of $17 \mathrm{~dB}$.

We obtained a highly nonlinear HF with a high SBS threshold by ensuring structural nonuniformity along its length during the fiber fabrication process through variation of the external fiber diameter [10]. The structural variation of the HF was not precisely specified in this instance. We simply switched off the active diameter feedback control ordinarily used when drawing fiber. This resulted in an HF with larger than normal diameter fluctuations. A cross-sectional scanning electron microscope (SEM) image of the HF used in this experiment is shown in the inset of Fig. 1. The core diameter is $\sim 1.2 \mu \mathrm{m}$ and the outer diameter is $\sim 150 \mu \mathrm{m}$. The measured loss was $190 \mathrm{~dB} / \mathrm{km}$ at $1550 \mathrm{~nm}$ and thus the effective length of the 15-m HF was only $11 \mathrm{~m}$. We measured the nonlinearity coefficient $\gamma$ to be $\sim 70( \pm 10) \mathrm{W}^{-1} \cdot \mathrm{km}^{-1}$, which is $\sim 40$ times higher than that of conventional dispersion shifted fibers (DSFs), using a direct $\mathrm{CW}$ measurement of the nonlinear phase shift suffered by a beat signal propagating in the fiber. The measurement procedure is fully described in [12]. The accurate derivation of the nonlinear coefficient $\gamma$ from the measured phase shift is dependent on measurements of the fiber loss and optical coupling. These measurements are somewhat more difficult to measure with accuracy using HF, and thus, the error quoted is somewhat larger than might be considered usual for the technique when applied to standard fiber types. Such a high nonlinearity allows for a reduction in the device length which is one of the key factors required in order to achieve broad-band wavelength conversion [7], [8]. The measured group velocity dispersion (GVD) of the $\mathrm{HF}$ is $\sim-30 \mathrm{ps} / \mathrm{nm} / \mathrm{km}$ at a wavelength of $1550 \mathrm{~nm}$. Due to the relatively short length of the fiber, and relatively narrow operating bandwidth of our measurement setup, we were unable to get a reliable measurement of the dispersion slope. Our best estimate from numerical simulations of the structure would be that the average zero-dispersion wavelength along the fiber would be located around $1550 \mathrm{~nm}$, although it should be appreciated that this is likely to vary along the fiber length due to the structural nonuniformity. The predicted average dispersion slope is approximately $-0.6 \mathrm{ps} / \mathrm{nm}^{2} / \mathrm{km}$. We then characterized SBS properties of the 15-m-long HF. We first measured the spontaneous Brillouin scattering lineshape of the 15-m HF using a hetrodyne-detection technique that relies upon beating the spontaneously scattered light with the pump beam [10]. Fig. 2(a) shows the measured spontaneous Brillouin spectrum. A non-Lorentzian lineshape comprising two distinct peaks with $\sim 75-\mathrm{MHz}$ frequency separation is clearly observed and results from the structural nonuniformity along the fiber length. The spontaneous Brillouin 3-dB bandwidth of one of the two peaks is seen to be approximately $50 \mathrm{MHz}$, which is over five times broader than would be expected for a silica fiber with a uniform cross-sectional profile [10]. The SBS threshold of the 15-m HF we used for wavelength conversion was estimated to be higher than $130 \mathrm{~mW}$ since we were unable to observe SBS due to the limited output power of our amplifier

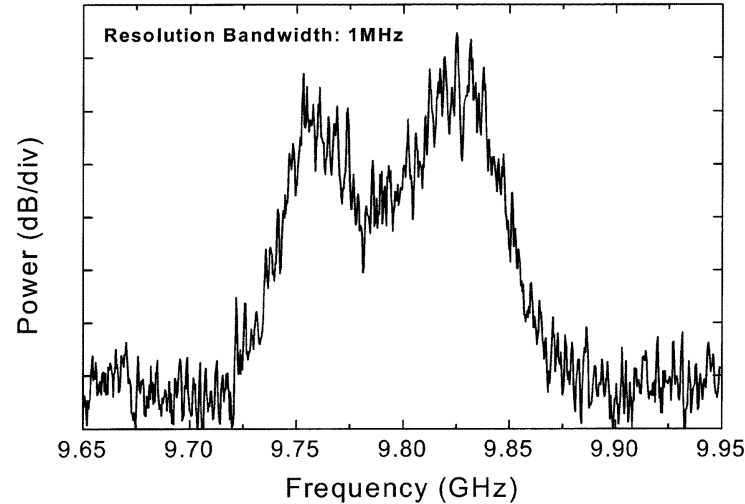

(a)

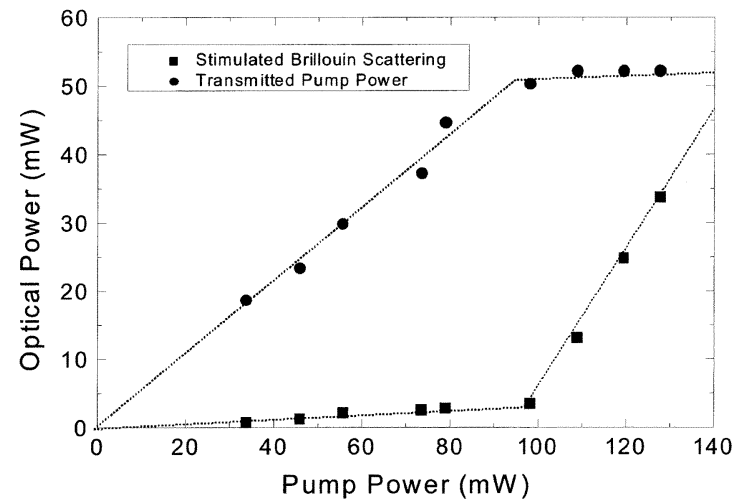

(b)

Fig. 2. (a) Radio frequency trace of the beat signals between spontaneous Brillouin scattered beam and the pump, using the 15-m-long HF described in Fig. 1(a). (b) SBS and transmitted pump power as a function of launched pump power, using a $20-\mathrm{m}$ length of nominally the same fiber.

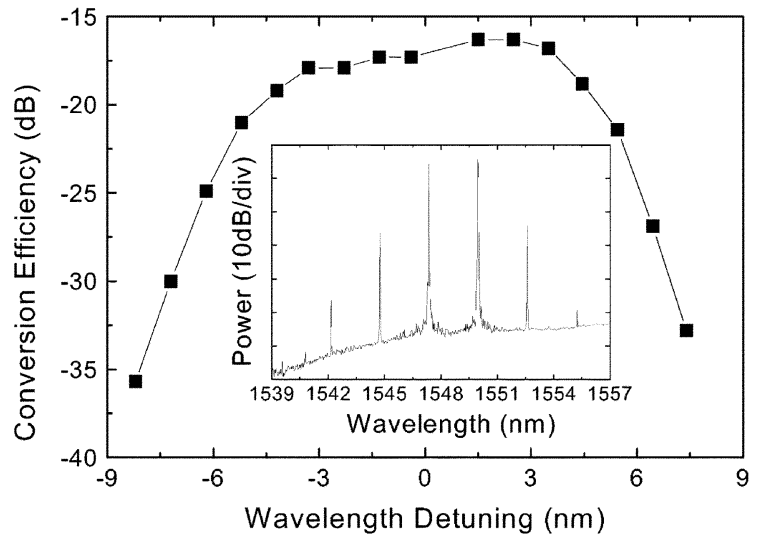

Fig. 3. Measured conversion efficiency versus wavelength detuning relative to a fixed signal wavelength of $1550 \mathrm{~nm}$. The inset shows the output FWM spectrum after the HF only (input signal at $\lambda=1550 \mathrm{~nm}$, pump at $\lambda=1547$ $\mathrm{nm})$.

(maximum pump power inside the HF: $\sim 130 \mathrm{~mW}$ ). The SBS threshold of a $20-\mathrm{m}$ length of nominally the same fiber was measured to be $\sim 100 \mathrm{~mW}$, as shown in Fig. 2(b), which is around two times higher than would be expected for a silica fiber with a uniform cross-sectional profile.

The HF output spectrum is shown in the inset of Fig. 3. The wavelengths of the initial signal and the pump beams are 1550 and $1547 \mathrm{~nm}$, respectively. A strong FWM wavelength converted 


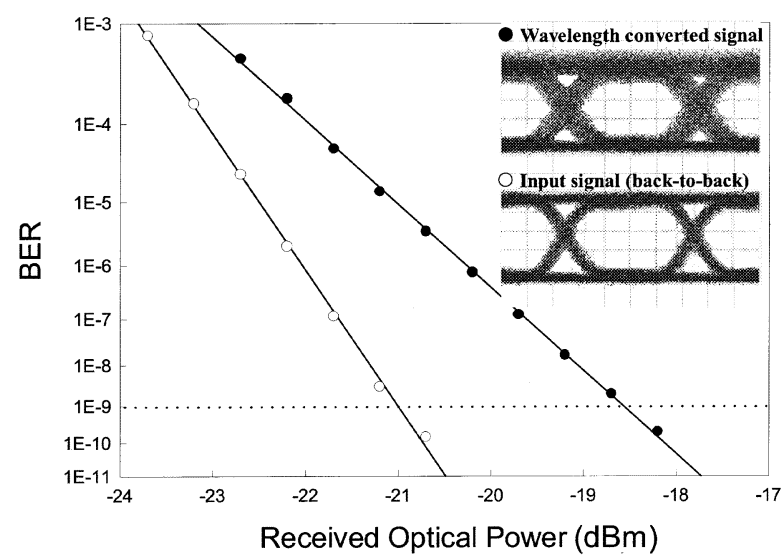

Fig. 4. Measured BER versus received optical power for wavelength conversion of a 10-Gb/s NRZ signal at a wavelength of $1544 \mathrm{~nm}$. Inset: Eye diagrams.

signal is clearly evident at a wavelength of $1544 \mathrm{~nm}$ despite the short fiber length used and both second- and third-order idler beams are also observable. After the HF, we used a compression-tunable two-stage apodized fiber Bragg grating (FBG) filter with a 3-dB bandwidth of $0.55 \mathrm{~nm}$ and a 30-dB bandwidth of $1.5 \mathrm{~nm}$ to efficiently suppress the input signal and pump beams and to pick out the wavelength converted signal [6]. A $\sim 40-\mathrm{dB}$ extinction ratio between the idler and the pump was achieved.

Fig. 3 shows the measured conversion efficiency, which is defined as the ratio of output wavelength-converted signal power to the input signal power. A maximum conversion efficiency of $-16 \mathrm{~dB}$ was achieved over a $3-\mathrm{dB}$ bandwidth of $\sim 10 \mathrm{~nm}$.

In order to analyze the system impact of using our HF-based FWM wavelength converter in practical WDM applications, we performed bit-error-rate (BER) measurements on the wavelength converted channel at $1544 \mathrm{~nm}$ (see the inset of Fig. 3). Fig. 4 shows both the measured BER and eye diagrams for both the 10-Gb/s NRZ input signal and wavelength converted output signal. We obtained error-free (BER $<10^{-9}$ ) wavelength conversion performance with a $\sim 2-\mathrm{dB}$ power penalty relative to that of the back-to-back input signal. The conversion efficiency will be a function of pump wavelength, and the BER performance will also depend on the pump and signal wavelengths since the HF is operated in a wavelength regime in which the dispersion slope is relatively high and the net GVD is small.

A small amount of timing jitter and intensity noise is observed from the eye diagrams, which we believe to be due to polarization coupling in this highly birefringent small core fiber. However, since the fiber had normal dispersion, no modulational-instability-induced intensity noise was observed [11]. In order to ensure the benefits of the use of normal dispersion fibers in the realization of this sort of nonlinear fiber devices, we performed similar FWM-based wavelength conversion experiments using an $\mathrm{HF}$ with an anomalous dispersion of $+10 \mathrm{ps} / \mathrm{nm} / \mathrm{km}$. The intensity noise, which can be attributed to modulational instability in anomalous dispersion regime, was so severe that BER measurements could not be performed.

\section{CONCLUSION}

We have experimentally demonstrated an HF-based tunable wavelength converter using FWM. Error-free wavelength conversion of $10 \mathrm{~Gb} / \mathrm{s}$ NRZ data signals over a 10-nm bandwidth was reliably achieved using just $15 \mathrm{~m}$ of highly nonlinear $\mathrm{HF}$ with a high SBS threshold. Although our HF-based FWM wavelength converter show slightly worse performance in terms of conversion efficiency and tuning bandwidth compared to the results achieved with highly nonlinear fiber [7], the use of a highly nonlinear HF with lower and flatter GVD should allow for further improvements. However, nonuniform HF technology offers significant advantages in terms of reduced device length, reduced power requirements, and ease of use since it eliminates the need for active schemes to reduce pump-power loss caused by Brillouin scattering.

\section{ACKNOWLEDGMENT}

The authors would like to thank M. Ibsen and M. R. Mokhtar for the use of their FBG and related help.

\section{REFERENCES}

[1] T. Hasegawa, E. Sasaoka, M. Onishi, M. Nishimura, Y. Tsuji, and M. Koshiba, "Novel hole-assisted lightguide fiber exhibiting large anomalous dispersion and low loss below $1 \mathrm{~dB} / \mathrm{km}$," in Proc. OFC, Anaheim, CA, Mar. 2001, Paper PD5.

[2] T. M. Monro, K. M. Kiang, J. H. Lee, K. Frampton, Z. Yusoff, R. Moore, J. Tucknott, D. W. Hewak, H. N. Rutt, and D. J. Richardson, "High nonlinearity extruded single-mode holey fibers," in Proc. OFC, Anaheim, CA, Mar. 2002, Paper PD1.

[3] Z. Yusoff, J. H. Lee, W. Belardi, T. M. Monro, P. C. Teh, and D. J. Richardson, "Raman effects in a highly nonlinear holey fiber: Amplification and modulation," Opt. Lett., vol. 27, pp. 424-426, 2002.

[4] J. H. Lee, P. C. The, Z. Yusoff, W. Belardi, M. Ibsen, T. M. Monro, and D. J. Richardson, "A holey fiber based nonlinear thresholding device for optical CDMA receiver performance enhancement," IEEE Photon. Technol. Lett., vol. 14, pp. 876-878, June 2002.

[5] L. K. Oxenløwe, A. I. Siahlo, K. S. Berg, A. Tersigni, A. T. Clausen, C. Peucheret, P. Jeppesen, K. P. Hansen, and J. R. Jensen, "A photonic crystal fiber used as a 160 to $10 \mathrm{~Gb} / \mathrm{s}$ demultiplexer," in Proc. OECC, Yokohama, Japan, July 2002, Paper PD1-4.

[6] J. H. Lee, Z. Yusoff, W. Belardi, M. Ibsen, T. M. Monro, and D. J. Richardson, "A tunable WDM wavelength converter based on cross phase modulation effects in normal dispersion holey fiber," IEEE Photon. Technol. Lett., vol. 15, pp. 437-439, Mar. 2003.

[7] O. Aso, S. Arai, T. Yagi, M. Tadakuma, Y. Suzuki, and S. Namiki, "Broadband four-wave mixing generation in short optical fiber," Electron. Lett., vol. 36, pp. 709-711, 2000.

[8] J. E. Sharping, M. Fiorentino, A. Coker, P. Kumar, and R. S. Windeler, "Four-wave mixing in microstructure fiber," Opt. Lett., vol. 26, pp. 1048-1050, 2001.

[9] J. Hansryd, F. Dross, M. Westlund, P. A. Antrekson, and S. N. Knudsen, "Increase of the SBS threshold in a short highly nonlinear fiber by applying a temperature distribution," J. Lightwave Technol., vol. 19, pp. 1691-1697, Nov. 2001.

[10] J. H. Lee, Z. Yusoff, W. Belardi, M. Ibsen, T. M. Monro, B. Thomsen, and D. J. Richardson, "Investigation of Brillouin effects in small-core holey optical fiber: Lasing and scattering," Opt. Lett., vol. 27, pp. 927-929, 2002.

[11] D. F. Grosz, C. Mazzali, S. Celaschi, A. Paradisi, and H. L. Fragnito, "Modulation instability induced resonant four-wave mixing in WDM systems," IEEE Photon. Technol. Lett., vol. 11, pp. 379-381, Mar. 1999.

[12] A. Boskovic, S. V. Chernikov, J. R. Taylor, L. Gruner-Nielsen, and O. A. Levring, "Direct continuous-wave measurement of $\mathrm{n}_{2}$ in various types of telecommunication fiber at $1.55 \mu \mathrm{m}$," Opt. Lett., vol. 21, pp. 1966-1968, 1996. 